\title{
Breast Cancer Treatment during the COVID-19 Pandemic
}

COVID-19 pandemic has challenged the prioritization of various diseases in all healthcare systems for humanity across the globe, and cancer patients are not an exception. Approximately, 160,000 new cases and 80,000 deaths are attributed to breast cancer annually and it is the most common cancer in women in India. ${ }^{[1]}$ In India, most of the patients with cancer need to travel a long distance to consult an oncologist and pursue cancer treatment. Further, such patients may be predisposed to COVID-19 infection during chemotherapy sessions in day-care centers. To reduce the infections among cancer patients and also to reduce the burden on existing infrastructure of healthcare, we formulated guidance.

\section{Adjuvant (Curative Intent)}

While surgery is the most definitive treatment for breast cancer, chemotherapy, hormonal agents, and radiation therapy are directed at reducing the risk of recurrence. However, as the survival benefit with chemotherapy is highly variable based on patient and tumor characteristics, it is imperative to suggest changes in the present recommendations.

In uncertain times of COVID-19 pandemic, it becomes important to balance between the benefits and risks associated with chemotherapy. It is important to estimate the benefit of such therapy while weighing the risks associated with COVID-19 infection and associated mortality.

Predict Online was used to determine the absolute benefit in 10-year overall survival of the third-generation chemotherapy in various subsets. ${ }^{[2]}$ Some assumptions were made to depict the representative characteristics as these are continuous variables. For instance, age was set at 50 years, grade 2 for hormone-positive, and grade 3 for triple-negative and human epidermal growth factor receptor 2 (HER2)-positive tumors. The tumor size was assumed as $2 \mathrm{~cm}$, and $5 \mathrm{~cm}$ for $\mathrm{T} 1$ and $\mathrm{T} 2$ categories, respectively. Likewise, the number of positive nodes were assumed to be 3, 6, and 10 for N1, N2 and N3, respectively. Ki-67 was presumed positive for all patients with HER2-positive and triple-negative breast cancers.

The mortality rate with COVID-19 is estimated to be around $2 \%-3 \%$ in overall population. However, the mortality increases with age and those more than 80 years of age had a high mortality rate of $21.9 \%$ [Table 1]..$^{[3,4]}$ A report of patients with cancer from Wuhan suggested higher risk of severe events (defined as the proportion of patients being admitted to the intensive care unit [ICU] requiring invasive ventilation, or death) compared with patients without cancer (seven [39\%] of 18 patients vs.
$124[8 \%]$ of 1572 patients; $P=0 \cdot 0003) \cdot{ }^{[4]}$ However, the limitation of this study was its small sample size (18 patients), and 12 of 18 patients did not receive any active therapy at the time of infection. There was a debate that the older age (median 63 years) rather than the history of cancer in these patients predisposed them to higher mortality. ${ }^{[5]}$

The benefit of adjuvant chemotherapy in various representative subsets is shown in Tables 2 and 3, respectively. We observed that the absolute benefit of chemotherapy highly varied from $2.6 \%$ to $15.5 \%$.

The benefit of chemotherapy and mortality from COVID-19 are dependent on various factors including age, preexisting comorbid conditions, tumor characteristics, and the demographic area of treatment. The incremental impact of COVID-19 on chemotherapy-related toxicity and mortality is not yet known. However, it is quite expected to be higher than normal. Standard recommendations for the prevention of COVID-19 must be followed. In addition, we suggest the following measures that can help in the management of patients with breast cancer.

\begin{tabular}{lcc}
\hline \multicolumn{2}{c}{ Table 1: Age-wise mortality rates with COVID-19 } \\
\hline Age (years old) & $\begin{array}{c}\text { Death rate all cases } \\
\text { in China (\%) }\end{array}$ & $\begin{array}{c}\text { Death rate in Italy } \\
\text { (\%) }\end{array}$ \\
\hline $80+$ & 14.8 & 20.2 \\
$70-79$ & 8.0 & 12.8 \\
$60-69$ & 3.6 & 3.5 \\
$50-59$ & 1.3 & 1.0 \\
$40-49$ & 0.4 & 0.4 \\
$30-39$ & 0.2 & 0.3 \\
\hline
\end{tabular}

Table 2: Benefit of adjuvant chemotherapy in hormonepositive breast cancer

\begin{tabular}{ccc}
\hline & Ki-67 positive (\%) & Ki-67 negative (\%) \\
\hline T & & \\
N0 & $2.6(1.9-3.1)$ & $2(1.5-2.4)$ \\
N1 & $6.1(4.5-7.5)$ & $4.9(3.6-6.0)$ \\
N2 & $8.3(6.1-10)$ & $6.8(5.0-8.4)$ \\
N3 & $10.4(7.6-13)$ & $8.7(6.4-11)$ \\
T2 & & \\
N0 & $6.6(4.9-8.1)$ & $5.3(3.9-6.5)$ \\
N1 & $12.8(9.3-16)$ & $11.1(8.1-14)$ \\
N2 & $15(11-19)$ & $13.7(9.9-17)$ \\
N3 & $15.6(11-20)$ & $15.2(11-19)$ \\
T3/T4 & & \\
N0 & $6.3(4.6-7.8)$ & $5.1(3.8-6.3)$ \\
N1 & $11.6(8.4-15)$ & $10.2(7.4-13)$ \\
N2 & $12.9(9.2-17)$ & $12.2(8.7-15)$ \\
N3 & $12.8(8.9-17)$ & $13(9.2-17)$ \\
\hline
\end{tabular}


Table 3: Benefit of adjuvant chemotherapy in triplenegative and human epidermal growth factor receptor 2-positive breast cancer

\begin{tabular}{lcc}
\hline & TNBC & HER2 \\
\hline T1 (assumed 2 cm) & & \\
N0 & $7(5.1-8.6)$ & $8.9(6.5-11)$ \\
N1 & $12.6(9.1-16)$ & $14.4(10-18)$ \\
N2 & $14.6(10-18)$ & $15.6(11-20)$ \\
N3 & $15.5(11-20)$ & $15.3(11-20)$ \\
T2 (assumed 5 cm) & & \\
N0 & $10.4(7.6-13)$ & $12.5(9.1-16)$ \\
N1 & $15.3(11-20)$ & $15.5(11-20)$ \\
N2 & $15.4(11-20)$ & $13.8(9.3-18)$ \\
N3 & $15.6(11-20)$ & \\
T3/T4 (size: 7 cm) & & \\
N0 & $12.2(8.9-15)$ & $14.2(10-18)$ \\
N1 & $15.6(11-20)$ & $14.3(9.8-19)$ \\
N2 & $14.1(9.6-19)$ & $11.1(7.2-15)$ \\
N3 & $11.5(7.5-16)$ & $7.5(4.7-11)$ \\
\hline TNBC
\end{tabular}

TNBC - Triple-negative breast cancer; HER2 - Human epidermal growth factor receptor 2

For patients with small-sized tumors upto $5 \mathrm{~cm}$, and those with limited nodal burden (0-3), a shorter course of trastuzumab for six months may be considered. In patients who have completed six months of therapy, further infusions of trastuzumab may be suspended after discussion. ${ }^{[6]}$ However, maintenance trastuzumab may be withheld completely in patients aged over 60 years, who are especially at a higher risk of complications from COVID-19 infection.

We suggest that a blanket statement on adjuvant chemotherapy in patients with breast cancer during COVID-19 cannot be made because of the wide range in absolute benefit of chemotherapy in different patients. Age and preexisting comorbid conditions along with tumor characteristics should be taken into account while making an informed decision with the patient.

Concerns regarding cardiac side effects must be taken into account as COVID-19 is known to bind to angiotensin-converting enzyme 2 receptors in the lower respiratory tracts of infected patients to gain entry into the lungs, ${ }^{[7]}$ and some reports of cardiac failure have been reported ${ }^{[8]}$ Although no data exist, it is likely that anthracyclines and HER2-blocking agents may increase the cardiac injury.

While routine use of growth factors is not recommended in 3 -weekly chemotherapy in breast cancer, this can reduce the duration of neutropenia and chances of febrile neutropenia and may be used, if feasible. Oral fluoroquinolone prophylaxis may be considered in those with higher risk of febrile neutropenia.

While delay of adjuvant chemotherapy beyond 90 days is associated with worse overall survival, we can consider delaying the start of adjuvant chemotherapy up to
12 weeks, which is likely to be the period when COVID-19 curve may start to decrease. For those, who are already on chemotherapy, a reassessment may be done to discuss the absolute benefit and continue chemotherapy if benefit outweighs the risk.

\section{Surgery and Radiotherapy}

The primary objective is to delay surgery till the pandemic is over, wherever feasible. This is to decrease the number of patients in postoperative wards and ICUs and decrease the utilization of limited number of personal protective equipment. Thus, locally advanced tumors may continue to start with neoadjuvant chemotherapy, while early breast cancer tumors may be initiated on antiestrogen therapy in hormone-positive breast cancers, chemotherapy in triple-negative breast cancer, and a combination of chemotherapy and targeted therapy in HER2-positive therapy. The society of surgical oncology has released the guidelines for surgical management during the pandemic and may be referred to for further details. ${ }^{[9]}$

Regarding radiotherapy, adjuvant treatment may be delayed up to 6 months because of multiple visits needed to administer such treatment. Further, hypofractionated radiotherapy may be preferred during this time to limit the number of hospital visits in institutions where conventional fractionation is used routinely. ${ }^{[10]}$ Moreover, accelerated partial breast irradiation (APBI) may also be used where the criteria are met to further limit the number of visits. ${ }^{[1]}$ Palliative radiation, if deemed necessary, must be delivered in least possible fractions, preferably on the same day of consultation and planning.

\section{Metastatic Disease (Palliative Intent)}

Regarding metastatic disease, those who are hormone positive must continue with hormonal therapy. Delaying the start of CDK4/6 inhibitors in patients with previously documented or high risk of neutropenia or febrile neutropenia may be considered. Second-line use of CDK $4 / 6$ inhibitors should be considered to reduce frequent hospital visits and monitor $\mathrm{CBC}$ during the initial 3 months. This may also reduce the incidence of febrile neutropenia. Hospital visits for zoledronic acid infusion must be postponed. It may be reasonable to defer 1-2 cycles of chemotherapy if the disease is stable and the burden of disease is low. Patients with significant disease burden may require continuation or early initiation of therapy.

Patients with triple-negative metastatic disease may consider switching to oral capecitabine, if the disease is relatively stable. Weekly chemotherapy may be converted to 3 weekly, and chemotherapy breaks for 4-6 weeks may be considered in those with relatively stable disease to tide over the peak of COVID-19 in India.

To conclude, uniform guidance on the treatment of patients with breast cancer during the COVID-19 pandemic is not 
feasible, due to the heterogeneity of disease. Individualized decisions are highly encouraged for next few weeks till the risk of acquiring COVID-19 infection significantly reduces. This statement may help in making decisions in this extraordinary time.

Telemedicine practices using voice/video calls, text messaging, or e-mails, which we otherwise discourage using during normal circumstances, are being utilized for social distancing. It may potentially be challenging or overwhelming in some settings, however, for oncologists. Hence, their judicious use is advisable.

The Government of India has issued the guidelines for teleconsultations. ${ }^{[12]}$ The appropriateness of telemedicine has been left at the discretion of a treating physician, based on patient's general condition and complexity of health issues. The physician should reveal his/her identity and the patient must provide consent for telemedicine if telemedicine is initiated by the physician (consent by parents or legal guardians in case of minors). If the patient initiates the telemedicine consultation, the consent is considered implied. The medical council registration of the physician must be displayed; a real-time consultation may be preferable over an asynchronous exchange of information. Drugs listed in Schedule $\mathrm{X}$ and any narcotics cannot be prescribed using any method of telemedicine. Further details may be retrieved from the telemedicine guidelines. ${ }^{[12]}$ It can be read and its recommendation may be included in a short paragraph.

\section{Atul Batra', Prashant Mehta ${ }^{2}$, Amol Patel ${ }^{3}$, Sainath Bhethanabhotla ${ }^{4}$, Bivas Biswas ${ }^{5}$, Raja Pramanik ${ }^{1}$, Chandan Krushna Das ${ }^{6}$}

${ }^{I}$ Department of Medical Oncology, All India Institute of Medical Sciences, New Delhi, India, '2Department of Medical Oncology, Hematoncology, and Bone Marrow Transplantation, Asian Institute of Medical Sciences,

Faridabad, Haryana, India, ${ }^{3}$ Malignant Diseases Treatment Centre, Army Hospital Research and Referral, New Delhi, India, ${ }^{4}$ Department of Medical Oncology, Krishna Institute of Medical Sciences, Hyderabad, Telangana, India, ${ }^{5}$ Department of Medical Oncology, Tata Medical Center, Kolkata, West Bengal, India, ${ }^{6}$ Department of Medical Oncology, PGI, Chandigarh, India

Address for correspondence: Dr. Atul Batra, Department of Medical Oncology, All India Institute of Medical Sciences, New Delhi, India. E-mail:batraatul85@gmail.com

Submitted: 28-Mar-2020 Revised: $10-\mathrm{Apr}-2020$ Accepted: 11-Apr-2020 Published: 05-May-2020

\section{References}

1. Globocan 2018: India Factsheet. India against Cancer; October, 2018. Available from: http://cancerindia.org.in/ globocan-2018-india-factsheet/. [Last accessed on 2020 Apr 07].
2. Predict Breast. Available from: https://breast.predict.nhs.uk/ tool. [Last accessed on 2020 Mar 23].

3. Team TNCPERE. The epidemiological characteristics of an outbreak of 2019 novel coronavirus diseases (COVID-19) China, 2020. CCDCW 2020;2:113-22.

4. Liang W, Guan W, Chen R, Wang W, Li J, Xu K, et al. Cancer patients in SARS-CoV-2 infection: A nationwide analysis in China. Lancet Oncol 2020;21:335-7.

5. Wang H, Zhang L. Risk of COVID-19 for patients with cancer. Lancet Oncol 2020;21:e181.

6. Earl HM, Hiller L, Vallier A-L, Loi S, McAdam K, Hughes-Davies L, et al. 6 versus 12 months of adjuvant trastuzumab for HER2-positive early breast cancer (PERSEPHONE): 4-year disease-free survival results of a randomised phase 3 non-inferiority trial. The Lancet 2019;393:2599-612.

7. Diaz JH. Hypothesis: Angiotensin-converting enzyme inhibitors and angiotensin receptor blockers may increase the risk of severe COVID-19. J Travel Med 2020. pii: taaa041.

8. Driggin E, Madhavan MV, Bikdeli B, Chuich T, Laracy J, Bondi-Zoccai G, et al. Cardiovascular considerations for patients, health care workers, and health systems during the coronavirus disease 2019 (COVID-19) pandemic. J Am Coll Cardiol 2020. pii: S0735-1097 (20) 34637-4.

9. Available from: http://Breast-Resource-DuringCOVID-19-3.30.20.pdf. [Last accessed on 2020 Apr 07].

10. Wang SL, Fang H, Song YW, Wang WH, Hu C, Liu YP, et al. Hypofractionated versus conventional fractionated postmastectomy radiotherapy for patients with high-risk breast cancer: A randomised, non-inferiority, open-label, phase 3 trial. Lancet Oncol 2019;20:352-60.

11. Correa C, Harris EE, Leonardi MC, Smith BD, Taghian AG, Thompson AM, et al. Accelerated partial breast irradiation: Executive summary for the update of an ASTRO evidence-based consensus statement. Pract Radiat Oncol 2017;7:73-9.

12. Available from: http://Telemedicine.pdf. [Last accessed on 2020 Apr 07].

This is an open access journal, and articles are distributed under the terms of the Creative Commons Attribution-NonCommercial-ShareAlike 4.0 License, which allows others to remix, tweak, and build upon the work non-commercially, as long as appropriate credit is given and the new creations are licensed under the identical terms.

For reprints contact: WKHLRPMedknow_reprints@wolterskluwer.com

\begin{tabular}{|l|l|}
\hline \multicolumn{2}{|c|}{ Access this article online } \\
\hline Quick Response Code: & Website: \\
& www.ijmpo.org \\
\cline { 2 - 2 } & DOI: \\
\hline
\end{tabular}

How to cite this article: Batra A, Mehta P, Patel A, Bhethanabhotla S, Biswas B, Pramanik R, et al. Breast cancer treatment during the COVID-19 pandemic. Indian J Med Paediatr Oncol 2020;41:135-7. 